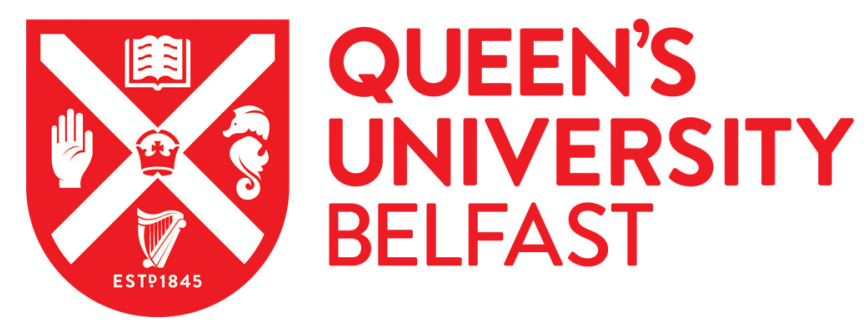

\title{
Retinal vascular oxygen saturation in response to a less extensive laser treatment in proliferative diabetic retinopathy
}

\author{
Vergmann, A. S., Torp, T. L., Kawasaki, R., Hestoy, D. H., Wong, T. Y., Peto, T., \& Grauslund, J. (2020). Retinal \\ vascular oxygen saturation in response to a less extensive laser treatment in proliferative diabetic retinopathy. \\ Acta Ophthalmologica. https://doi.org/10.1111/aos.14727
}

Published in:

Acta Ophthalmologica

Document Version:

Peer reviewed version

Queen's University Belfast - Research Portal:

Link to publication record in Queen's University Belfast Research Portal

Publisher rights

Copyright 2020 Wiley. This work is made available online in accordance with the publisher's policies. Please refer to any applicable terms of use of the publisher.

\section{General rights}

Copyright for the publications made accessible via the Queen's University Belfast Research Portal is retained by the author(s) and / or other copyright owners and it is a condition of accessing these publications that users recognise and abide by the legal requirements associated with these rights.

Take down policy

The Research Portal is Queen's institutional repository that provides access to Queen's research output. Every effort has been made to ensure that content in the Research Portal does not infringe any person's rights, or applicable UK laws. If you discover content in the Research Portal that you believe breaches copyright or violates any law, please contact openaccess@qub.ac.uk. 
Title page

Title:

Retinal vascular oxygen saturation in response to a less extensive laser treatment in proliferative diabetic retinopathy

Authors:

Anna Stage Vergmann ${ }^{1,2,4}$, Thomas Lee Torp ${ }^{1,2}$, Ryo Kawasaki ${ }^{2,5}$, Danjal Hofgaard Hestoy ${ }^{1}$, Tien Yin Wong ${ }^{6,7}$, Tunde Peto ${ }^{2,8}$ and Jakob Grauslund ${ }^{1,2,3}$

${ }^{1}$ Department of Ophthalmology, Odense University Hospital, Odense, Denmark

${ }^{2}$ Research Unit of Ophthalmology, Department of Clinical Research, Faculty of Health Science, University of Southern Denmark, Odense, Denmark

${ }^{3}$ Steno Diabetes Center Odense, Odense University Hospital, Odense, Denmark

${ }^{4}$ OPEN, Open Patient data Explorative Network, Odense University Hospital, Odense, Denmark

${ }^{5}$ Artificial Intelligence Center for Medical Research and Application, Osaka University Hospital, Osaka, Japan

${ }^{6}$ Singapore Eye Research Institute, Singapore National Eye Centre, Singapore

${ }^{7}$ Duke-NUS Medical School, National University of Singapore, Singapore

${ }^{8}$ School of Medicine, Dentistry and Biomedical Sciences, Queen's University, Belfast, Northern Ireland, United Kingdom

Corresponding author:

Anna Stage Vergmann

Department of Ophthalmology, Odense University Hospital

Kloevervaenget 5, entrance 132

DK-5000 Odense C, Denmark

Phone:+45 65411682

E-mail: anna.stage.vergmann@rsyd.dk 


\section{Abstract}

Purpose: The purpose of this study was to evaluate the association between retinal laser burden and vascular oxygen saturation in patients with proliferative diabetic retinopathy (PDR) treated with different extent of retinal laser.

Methods: The study was a prospective, interventional study of patients with treatment-naïve PDR. Patients were treated with navigated retinal laser (Navilas ${ }^{\circledR}, \mathrm{OD}-\mathrm{OS} \mathrm{GmbH}$, Teltow, Germany) in different doses. Retinal oximetry was obtained at baseline (BL) prior to laser and after six mon ths (M6). Patients were divided into three groups according to total laser spots applied: $<1500$ spots (Group 1), 1500-2000 spots (Group 2), and >2000 spots (Group 3).

Results: We included 33 eyes of 28 patients with treatment-naïve PDR. The groups did not differ according in $\mathrm{BL}$ characteristics. Between BL and M6, retinal arteriolar oxygen saturation did not change but retinal venular oxygen saturation (median with interquartile range) decreased in Groups 1 and 2 (1: $65.5 \pm 8.8 \%$ vs. $60.5 \pm 9.5 \%, p=0.04 ; 2: 65.3 \pm 7.3 \%$ vs. $63.0 \pm 13.5 \%, p=0.04)$. Focal retinal venular oxygen saturation, located to quadrants with retinal neovascularization, decreased in Group 2 from BL to M6 $(67.5 \pm 13.3 \%$ vs. $61.5 \pm 8.8 \%, p=0.04)$. Retinal venular diameter decreased from BL to M6 in Group 1 (174.5 $\pm 15.3 \mu \mathrm{m}$ vs. $165.1 \pm 28.7 \mu \mathrm{m}, \mathrm{p}=0.01)$.

Conclusions: In this study of patients with treatment-naïve PDR, we showed that a less extensive laser treatment caused a reduction in retinal venular oxygen saturation and diameter six months after treatment. Our results suggest that less extensive laser treatment may be sufficient to improve the retinal metabolic environment conducive to PDR regression.

Key words: oximetry, proliferative diabetic retinopathy, laser treatment, oxygen saturation 


\section{Introduction:}

Diabetic retinopathy (DR) is the most common microvascular complication in diabetes (Grauslund et al. 2009) and a major cause of blindness (Grauslund et al. 2009; Leasher et al. 2016; Ting et al. 2016). DR emerges from hypoxia caused by retinal neurovascular damage, and proliferative diabetic retinopathy (PDR) is the ocular end-stage of the disease (Wong et al. 2016). PDR is characterized by the formation of retinal neovascularization that can lead to vitreous haemorrhage or tractional retinal detachment, if not treated in time. The standard treatment of PDR is panretinal photocoagulation (PRP) as defined by The Diabetic Retinopathy Study Group (The Diabetic Retinopathy Study Research Group 1981), where treatment is applied globally with coverage of the entire retina except the centre, including macula, the opticnerve, largest blood vessels, and the proliferative area. Other treatment strategies have been proposed, such as subthreshold microsecond laser (Jhingan et al. 2018) and 'light PRP' (Bandello et al. 2001). While the exact mechanism of PRP is still unknown, it has been proposed that retinal laser is absorbed by the pigmented cells, which in terms destroys the retinal pigment epithelium and photoreceptors, hereby decreasing the hypoxic load(Stefansson 2006). Furthermore, laser treatment facilitates choroidal oxygen diffusion through laser scars that makes more oxygen available in the inner retina (Stefansson 2006; Wong et al. 2016). However, clinical demonstration of these hypothetical mechanisms after PRP is challenging.

Retinal oximetry is a non-invasive way to measure the oxygen saturation in the retinal vessels. It can be used to detect retinal hypoxia and disturbances in the retinal oxygenation. It has previously been shown that increasing levels of DR is associated with increasing retinal venular oxygen saturation, while retinal arteriolar diameter is decreased in patients with PDR (Hardarson \& Stefánsson 2012; Guduru et al. 2016; Blair et al. 2017; Rilvén et al. 2017). Furthermore, it has been demonstrated that retinal venular oxygen saturation is stabilised in patients with PDR successfully treated with PRP and that retinal venular diameter is increased in patients with non-proliferative DR (Blair et al. 2017; Torp et al. 2018). Previous results on retinal arteriolar oxygen saturation have shown correlation with an increase with increasing degrees of DR and ischemia (Guduru et al. 2016) while others only found a difference in the latest stages of DR (Khoobehi et al. 2013).

Thus, while the association between DR and retinal venular oxygen saturation has previously been investigated (Guduru et al. 2016; Blair et al. 2017; Rilvén et al. 2017), previous studies have not investigated if a certain amount of retinal laser is needed to induce alte rations in the retinal metabolism that leads to PDR regression and stability. This is clinically important as too little laser may not be sufficient while too much laser is associated with adverse side effects. Therefore, the purpose of this study was to evaluate the association between retinal laser PRP treatment and retinal oxygen saturation in patients with treatmentnaïve PDR treated with different extent of retinal laser. We hypothesised that a less extensive laser burden would cause less damage on the retinal tissue but is sufficient to lead to improved retinal metabolic condition that will ultimately lead to PDR regression and stabilization.

\section{Methods:}

Study design and population 
This study is a sub study of a six-month 1:1 randomized controlled trial (RCT) which included 33 eyes of 28 patients with treatment-naïve PDR that were referred to Odense University Hospital, Odense, Denmark between June 1st, 2017 and February 1st, 2019 (Vergmann et al. 2020). Patients were either randomized to navigated retinal laser treatment (Navilas ${ }^{\circledR} 532 \mathrm{~nm}, \mathrm{OD}-\mathrm{OS} \mathrm{GmbH}$, Teltow, Germany) performed as standard or individualized PRP. Randomization was performed by Research Electronic Data Capture (REDCap) database under Open Patient data Explorative Network (OPEN). To ensure the same degree of ischemic disease, the two groups were balanced in relation to the number of retinal quadrants with proliferations. Inclusion criteria were diabetes mellitus (type 1 and 2), newly diagnosed, untreated PDR in one eye (possibility of inclusion of both eyes if bilateral PDR, in which case randomization would be with one eye in each group). We excluded patients with diabetic macular oedema (DMO) in the affe cted eye (central subfields thickness $>300 \mu \mathrm{m}$ ), age $<18$ years, pregnancy and/or blurry opticmedia that could prevent PRP or interfere with the quality of the retinal images taken. Patients were blinded to their treatment arm(s).

Treatment efficacy (progression of PDR) and side-effects (visual field, dark adaptation and retinal quality of life) were defined as main outcomes of the trial. Given that the main outcomes did not differ between patients with progression and patients without progression, eyes of both groups were pooled for the present study. Due to spontaneous vitreous haemorrhage and progression in cataract during follow -up, the retinal image quality was affected, thus the sub study is smaller than the original study.

\section{Panretinalphotocoagulation}

In the RCT, standard PRP were localized to all four retinal quadrants and individualized PRP was localized to retinal quadrants with retinal neovascularization only. Treatment was performed at baseline (BL) and supplemented if needed at month three (M3) and / or month six (M6). The treatment was executed in two sessions at BL in both groups. A Mainster 165 PRP contact lens was used, and the patients were given a local anaesthetic (oxybuprocaine hydrochloride $0.4 \%$ ) prior to treatment. During treatment, the following settings were used; spot size: $390 \mu \mathrm{m}$, pulse duration: $30 \mathrm{~ms}$, and power was adjusted to $280 \mathrm{~mW}$ and adjusted until a greyish-white indication was present on the retina. Supplemental PRP treatment were given at follow-up (M3 or M6) if progression of PDR were found by the grader (subjective assessed by ophthalmoscopy, wide-field fundus photo, and ultra-widefield photo and fluorescens angiography (FA) (Optos, Dunfermline, United Kingdom)). Progression was defined as increasing areas of neovascularization, increasing FA leakage, or vitreous haemorrhage at follow-up M3 or M6. Patients were divided into three groups according to number of retinal laser spots applied: $<1500$ spots ("light", Group 1), 1500-2000 spots ("moderate", Group 2), and $>\mathbf{2 0 0 0}$ spots ("extensive", Group 3). It should be noted all groups received a less extensive laser treatment, compared to the standard Early Treatment Diabetic Retinopathy Study protocol (Early Treatment Diabetic Retinopathy Study Research Group 1987) with both smaller spot size, pulse duration and fewer spots applied in Groups 1 and 2.

\section{Visualacuity and clinical examinations}

Demographicinformation, a full medical history, height, and weight (BMI) were collected at BL. At BL and M6, we measured best corrected visual acuity (BCVA), using the Early Treatment Diabetic Retinopathy Study (ETDRS) chart, intraocular pressure (Goldmann, Haag-Streit, Bern, Switzerland), brachial arterial blood pressure (Omron 705CP, Hoofddorp, The Netherlands). Venous blood samples of hemoglobin $A_{1 c}$ 
(HbA1c) were drawn. Spectral domain (SD) optical coherence tomography (OCT) (Topcon, Tokyo, Japan) and retinal oximetry (Oxymap model T1, Oxymap, software V.2.4.2, Reykjavik, Iceland) was performed, also at BL, M3 and M6. The patients underwent a standard ophthal mic examination including slit lamp examination performed in mydriasis with tropicamide $10 \mathrm{mg} / \mathrm{mL}$ and phenyl ephedrine $10 \%$, and wide-field fundus photo and FA were performed at BL, M3 and M6.

\section{Retinal oximetry}

Oxymap T1 was used to obtain retinal oxygen saturation by 50-degree optic disc centred images at BLand M6. Description of equipment and technique of image-capturing can be found elsewhere (Geirsdottir et al. 2012). Grading of all images were performed by a trained grader following a prespecified grading protocol. A circle was manually placed 20-30 pixels from the edge of the optic disc to avoid faulty measurements due to reflection from the optic disc and the retinal nerve fibre layer. A second circle was automatically generated measuring three times the diameter of the inner circle and the oxygen saturation measurements were done between the two circles. The widest possible arteriole and venule (50-200 pixels) was chosen in each quadrant and automatically traced. If the length of one of the chosen vessels was less than 50 pixels from the inner circle to the first branching point, the first branch was selected for tracing. At follow up, the same vessel segments were selected for tracing. Overall arteriolar and venular saturation are presented in this article and are defined as the mean oxygen saturation across the four quadrants. Focal arteriolar and venular retinal saturation were defined as the local oxygenation in only the treated quadrant(s). Retinal arteriolar and venular diameter from the oximetry gradings was analysed.

\section{Ethical considerations}

All patients participated on the basis of informed and written consent and were informed that they at any time could withdraw from the study without justification. If there were other conditions requiring treatment in connection with the treatment or examinations, patients were referred for appropriate treatment. The study was conducted in accordance with the Helsinki Declaration II and in accordance with good clinical practice. The project was approved by the Research Ethics Committee of Southern Denmark (Project-ID: S-20160168) and by The Danish Data Protection Agency. The full trial protocol can be found at https://clinicaltrials.gov (identifier: NCT03113006) as the project was registered online prior to initiation.

\section{Statistics}

Data are presented as median with interquartile range (IQR) interval. A mixed model including nested random effects on a patient and eye-within-patient level was used to analyse differences between groups and between time points. Model validation was performed by visual inspection of residuals, fitted values and random effect estimates. Pearson's correlation $r$ was used to detect correlation between laser spots and arterial and venular oxygen saturation at M6. Statistical differences were tested at a 0.05 level. Statistical analysis was performed using STATA Intercooled version 16 (StataCorp LLC, College Station, TX, USA).

\section{Results:}


A total of 33 eyes of 28 patients were included. As presented in Table 1, patients in Groups 1-3 did not differ according to age, sex, type 1 diabetes, duration of diabetes, $\mathrm{HbA1}$ cor best corrected visual acuity. Median (with IQR) power applied in each group was as follows; Group 1; $4.409 \pm 1.9 \mathrm{~J}$, Group 2: $6.626 \pm 3.8 \mathrm{~J}$, Group 3: $8.292 \pm 2.9 \mathrm{~J}, \mathrm{p}=0.002$. Median (with IQR) laser spots applied was as follows; Group 1: $1040 \pm 478$, Group 2: $1798 \pm 540$, Group 3: $2392 \pm 608, p<0.001$. Image quality did not differ between the groups (Table 2). There was no direct relationship between progression vs. non -progression and difference in retinal venular saturation from BL to M6 (Figure 1) or correlation ( $r$ ) between total laser s pots and overall oxygen saturation (arteriolar: $0.22, p=0.22$; venular: $0.29, p=0.10$; arterio-venous difference (AVD): $-0.30, p=0.08$ ).

\section{Global retinal vascularoxygen saturation and vessel diameter}

From BL to M6, retinal arteriolar oxygen saturation did not alter in any of the groups (Table 2 and Figure 2). Retinal venular oxygen saturation (mean) decreased during follow-up in Groups 1 and 2 (1: $65.5 \pm 8.8 \%$ vs. $60.5 \pm 9.5 \%, p=0.04 ; 2: 65.3 \pm 7.3 \%$ vs. $63.0 \pm 13.5 \%, p=0.04$ ) but not in Group 3. Mean AVDincreased in Group 1 during follow up ( $31.9 \pm 5.8$ vs. $35.9 \pm 13.0, p=0.03$ ) but no differences werefound in Groups 2 and 3 (Table 2 and Figure 3). Mean retinal arteriolar diameter did not statistically change at follow up in any of the groups. Mean retinal venular diamete r decreased during follow-up in Group 1 (174.5 $\pm 15.3 \mu \mathrm{m}$ vs. 165.1 $\pm 28.7 \mu \mathrm{m}, \mathrm{p}=0.01$ ) but did not change in Groups 2 and 3 (Table 3).

\section{Focal retinal oxygen saturation}

For patients with retinal neovascularization in one, two or three retinal quadrants, focal ven ular oxygen saturation decreased during follow up in Group 2 (67.5 $\pm 13.3 \%$ vs. $61.5 \pm 8.8 \%, p=0.04$ ), but not in Groups 1 and 3 (Table 4). No changes were seen in the retinal arteriolar oxygen saturation.

\section{Discussion:}

In this study of patients with treatment-naïve PDR, we demonstrated a decreasing retinal venular oxygen saturation in patients receiving lower amounts of retinal laser treatment. Our results suggest that a less extensive laser application exerts a demonstratable improvement in the retinal metabolic condition that is conducive to PDR regression and stabilization.

We investigated the effect of total retinal laser spots on retinal oxygen saturation and found that patients in Groups 1 ("light") and 2 ("moderate") had an observable decrease in retinal venular oxygen saturation 6 months after laser. Retinal blood flow is disturbed in patients with DR with presence of capillary nonperfusion leading to areas with ischemia. Another common phenomenon in DR is the shunting of blood to circumvent areas with vascular occlusion between venules and arterioles, where retinal oxygen saturation in arteriolar-venous and venous-venous shunts is increased compared to the retinal venular saturation (Petersen \& Bek 2019). When treating with retinal laser, areas in between the large vessels where shunting is present is ablated. The ablation of areas with shunting might have caused less shunting of blood from the arteriolar to the venular retinal vessels and reduced the retinal venular oxygen saturation. Furthermore, AVD increased in Group 1, as a result of the decreased retinal venular oxygen saturation. AVD can be interpreted as a an marker of oxygen-consumption in the tissue (Hardarson \& Stefánsson 2012) indicating increased oxygen-consumption was present for Group 1. 
A decrease of retinal venular oxygen saturation was not found in Group 3 ("extensive"). However, th is group started at a lower retinal venular and arteriolar oxygen saturation compared to the other two groups and a small decrease was seen from BL to M6. Even though the lower starting saturation was not statistically significant different from the two other groups, the low starting point could have been the reason that no differences was observed in Group 3, rather than the less extensivelaser approach. However, a bigger study cohort is needed to confirm this.

A corresponding decrease, as in retinal venular oxygen saturation, was observed in retinal venular diameter in the "light" laser group (Group 1). Previous studies have shown that blood flow in the retinal vessels is reduced after PRP (Oswald et al. 1985; Fujio et al. 1994). The decrease in diameter in this group could indicate reduced blood flow as an expression of less oxygen demand by the tissue. The fact that the decrease in vessel diameter was only present in Group 1 could indicate that this group especially had a good response to the less extensive laser treatment.

Oxygen saturation has shown to increase with increasing degree of DR (Rilvén et al. 2017). This has been explained by a combination of capillary non-perfusion, arterio-venular shunting, thickening of capillary walls, and greater oxygen affinity for glycosylated haemoglobin. This leads in turn to poor distribution and decreased consumption of oxygen in the retinal tissue. Conversely, in patients that are treated successfully with PRP, retinal venular oxygen saturation decreases (Torp et al. 2018) supporting evidence of increased oxygen consumption. In relation to this the decrease in retinal venous oxygen saturation and diameter in the group that received less extensive laser treatment in this study could imply successful treatment similar to that of Torp et al., but also with favourable anatomic outcome because of the less destructive nature. This is in line with previous findings of ind ividualised PRP of PDR (localized to affected quadrants) being as efficient as standard PRP (Vergmann et al. 2020). Even though changes in retinal venular oxygen saturation was not directly correlated with progression/non-progression in this study, the sample size was small and a trend towards a higher amount of laser spots and an increase in retinal venular oxygen saturation was observed.

Our findings of a decrease in retinal venular oxygen saturation are partly in conflict with a previous study. This study found an increase in retinal venular oxygen saturation in patients with PDR 3 months after treatment with PRP (Jørgensen \& Bek 2014). They applied a mean of 2232 spots. To compare, we did not find any differences in the retinal venular oxygen saturation in the group that received $>2000$ spots. This suggests that a treatment over a certain point initiate other mechanisms that either stabilises or switches the retinal blood flow. However, we had a longer follow-up time (6 months) which could have given the opportunity forfurther changes.

There were some limitations to this study. Given the nature of the study, it was only possible to include a small sample of patients followed for six months. Additionally, we did not have information available with respect to the exact stage of cataract as well as number of pseudo phakic eyes, which is known to influence the retinal oximetry (Della Vol pe Waizel et al. 2020). Furthermore, for ethical reasons, we could not include an untreated group of patients with PDR. However, the prospective st udy design made it possible to observe temporal changes within and between eyes as stratified for amount of laser treatment. Fora further study it could also be interesting to examine the effect of extent and area of ischemia to see how this affects retinal oxygenation. 
In conclusion, in this prospective study of patients with treatment-naïve PDR, we showed that a less extensive laser treatment caused a decrease in retinal venular oxygen saturation and diameter 6 months after treatment. No differences were found between patients with progression or non-progression of PDR. Our results suggest that less extensive laser treatment, with less damage of the retina, results in a measurable improvement in the retinal metabolic conditions that is associated with PDR regression and stabilization. The results should be interpreted with caution because of a small sample size.

\section{Acknowledgements}

We would like to acknowledge VELUX FONDEN and the PhD Foundation at Odense University Hospital for the financial support for this study. 
Bandello F, Brancato R, Menchini U, Virgili G, Lanzetta P, Ferrari E \& Incorvaia C (2001): Light Panretinal Photocoagulation (Lprp) Versus Classic Panretinal Photocoagulation (Cprp) in Proliferative Diabetic Retinopathy. Semin Ophthalmol 16: 12-18.

Blair NP, Wanek J, Felder AE, Joslin CE, Kresovich JK, Lim JI, Chau FY, Leiderman Y \& Shahidi M (2017): Retinal Oximetry and Vessel Diameter Measurements with a Commercially Available Scanning Laser Ophthal moscope in Diabetic Retinopathy. Investigative ophthalmology \& visual science 58: 55565563.

Della Volpe Waizel M, TürkseverC\& Todorova MG (2020): Influence of Cataract Light Scatters on Retinal Vessel Oxygen Saturation. Acta Ophthalmol 98: e56-e62.

Early Treatment Diabetic Retinopathy Study Research Group (1987): Techniques for Scatter and Local Photocoagulation Treatment of Diabetic Retinopathy: Early Treatment Diabetic Retinopathy Study Report No. 3. International Ophthalmology Clinics 27: 254-264.

Fujio N, Feke GT, Goger DG \& McMeel JW (1994): Regional Retinal Blood Flow Reduction Following Half Fundus Photocoagulation Treatment. The British Journal of Ophthal mology 78: 335-338.

Geirsdottir A, Palsson O, Hardarson SH, Olafsdottir OB, Kristjansdottir JV \& Stefánsson E(2012): Retinal Vessel Oxygen Saturation in Healthy Individuals. Investigative Ophthalmology \& Visual Science 53: 5433-5442.

Grauslund J, Green A \& Sjolie AK (2009): Blindness in a 25-Year Follow-up of a Population-Based Cohort of Danish Type 1 Diabetic Patients. Ophthalmology 116: 2170-2174.

Guduru A, Martz TG, Waters A, Kshirsagar AV \& Garg S (2016): Oxygen Saturation of Retinal Vessels in All Stages of Diabetic Retinopathy and Correlation to Ultra-Wide Field Fluorescein Angiography. Investigative Ophthalmology \& Visual Science 57:5278-5284.

Hardarson SH \& Stefánsson E (2012): Retinal Oxygen Saturation Is Altered in Diabetic Retinopathy. British Journal of Ophthalmology 96: 560.

Jhingan M, Goud A, Peguda HK, Khodani M, Luttrull JK \& Chhablani J (2018): Subthreshold Microsecond Laser for Proliferative Diabetic Retinopathy: A Randomized Pilot Study. Clin Ophthalmol 12: 141145.

Jørgensen C \& Bek T (2014): Increasing Oxygen Saturation in Larger Retinal Vessels after Photocoagulation for Diabetic Retinopathy. Investigative Ophthalmol ogy \& Visual Science 55: 5365-5369.

Khoobehi B, Firn K, Thompson H, Reinoso M\& Beach J (2013): Retinal Arterial and Venous Oxygen Saturation Is Altered in Diabetic Patients. Investigative Ophthalmology\& Visual Science 54: 71037106.

Leasher JL, Bourne RRA, Flaxman SR, Jonas JB, Keeffe J, Naidoo K, Pesudovs K, Price H, White RA, Wong TY, Resnikoff S \& Taylor HR (2016): Global Estimates on the Number of People Blind or Visually Impaired by Diabetic Retinopathy: A Meta-Analysis from 1990 to 2010. Diabetes Care 39: 1643.

Oswald B, Vilser W, Oswald H, Jütte A, Schweitzer D, Königsdörffer E, Deufrains A \& Dietze U(1985): Measurement of Flow-Physiologic Parameters of Retinal Blood Circulation in Type 1 and 2 Diabetics before and after Photocoagulation. Graefe's Archive for Clinical and Experimental Ophthalmology 223: 154-157.

Petersen L\& Bek T (2019): The Oxygen Saturation in Vascular Abnormalities Depends on the Extent of Arteriovenous Shunting in Diabetic Retinopathy. Investigative Ophthalmology \& Visual Science 60: 3762-3767.

Rilvén S, Torp TL \& Grauslund J (2017): Retinal Oximetry in Patients with Ischaemic Retinal Diseases. Acta Ophthalmologica 95: 119-127.

Stefansson E (2006): Ocular Oxygenation and the Treatment of Diabetic Retinopathy. Surv Ophthalmol 51: 364-380.

The Diabetic Retinopathy Study Research Group (1981): Photocoagulation Treatment of Proliferative Diabetic Retinopathy - Clinical Application of Diabetic Retinopathy Study (Drs) Findings. Drs Report Number 8. American Academy of Ophthalmology. 
Ting DSW, Cheung GCM \& Wong TY (2016): Diabetic Retinopathy: Global Prevalence, Major Risk Factors, Screening Practices and Public Health Challenges: A Review. Clinical \& Experimental Ophthalmology 44: 260-277.

Torp TL, Kawasaki R, Wong TY, Peto T \& Grauslund J (2018): Changes in Retinal Venular Oxygen Saturation Predict Activity of Proliferative Diabetic Retinopathy 3 Months after Panretinal Photocoagulation. The British Journal of Ophthalmology 102: 383-387.

Vergmann AS, Nguyen TT, Lee Torp T, Kawasaki R, Wong TY, Peto T \& Grauslund J (2020): Efficacy and Side Effects of Individualized Panretinal Photocoagulation. Ophthalmol Retina 4:642-644.

Wong TY, Cheung CMG, Larsen M, Sharma S \& Simó R (2016): Diabetic Retinopathy. Nature Reviews Disease Primers 2: 16012. 
Figure legends-Figure 1:

Scatter plot of the relationship between no (number) of total laser spots given and difference in retinal venular oxygen saturation (\%) from baseline to month 6 . The blue dots represent patients with progression of proliferative diabetic retinopathy (PDR), and the red dots represent patients with no progression of PDR. 
Figure legends-Figure 2:

A plot to illustrate the retinal arteriolar oxygen saturation before and six months after treatment divided by the treatment groups (group 1 (<1500 spots), group 2 (1500-2000 spots) and group 3 (>2000 spots)). 
Figure legends -Figure 3:

A plot to illustrate the retinal venular oxygen saturation before and six months after treatment divided by the treatment groups (group 1 (<1500 spots), group 2 (1500-2000 spots) and group 3 (>2000 spots)). 


\section{Figure 1:}

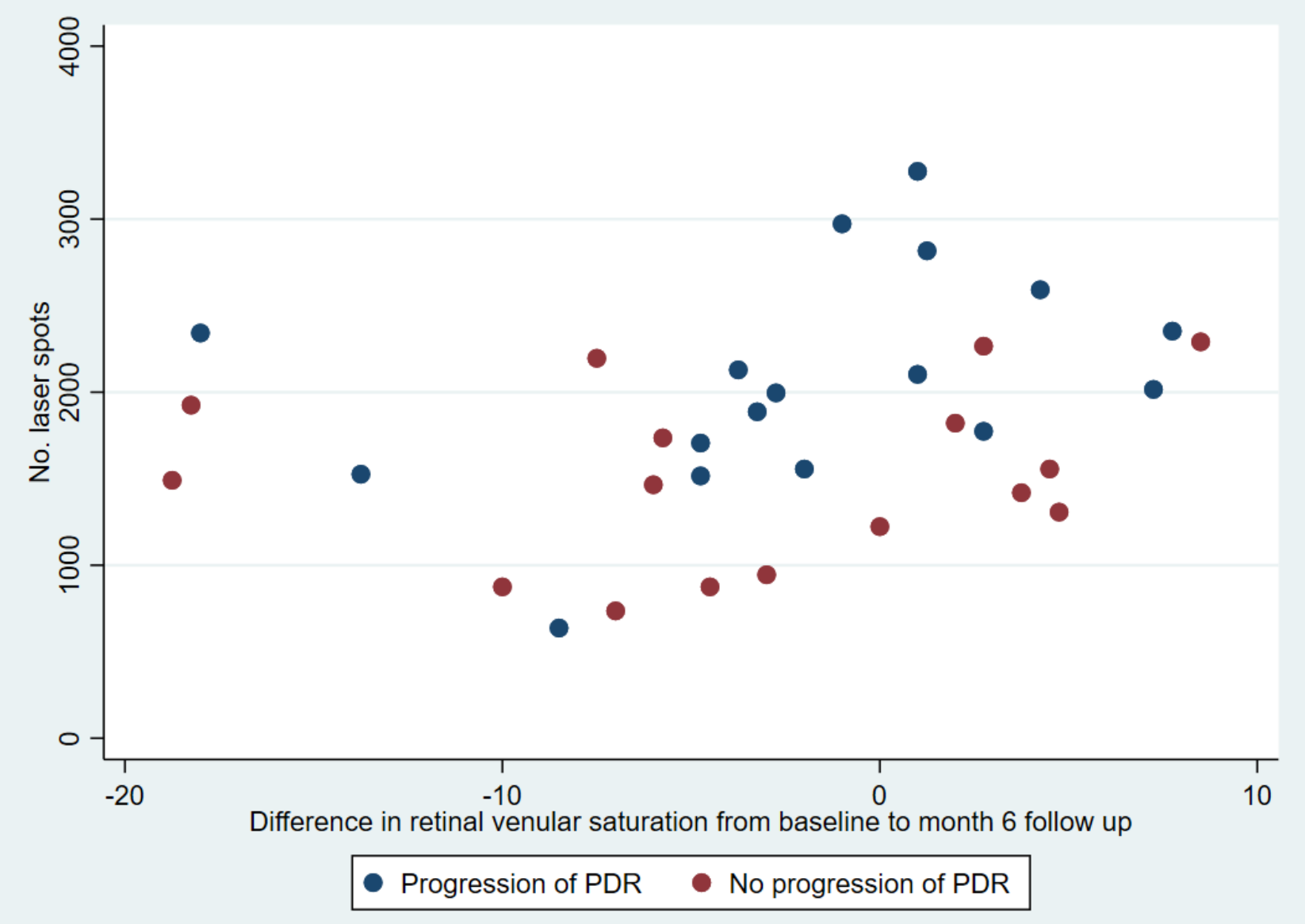


Figure 2:

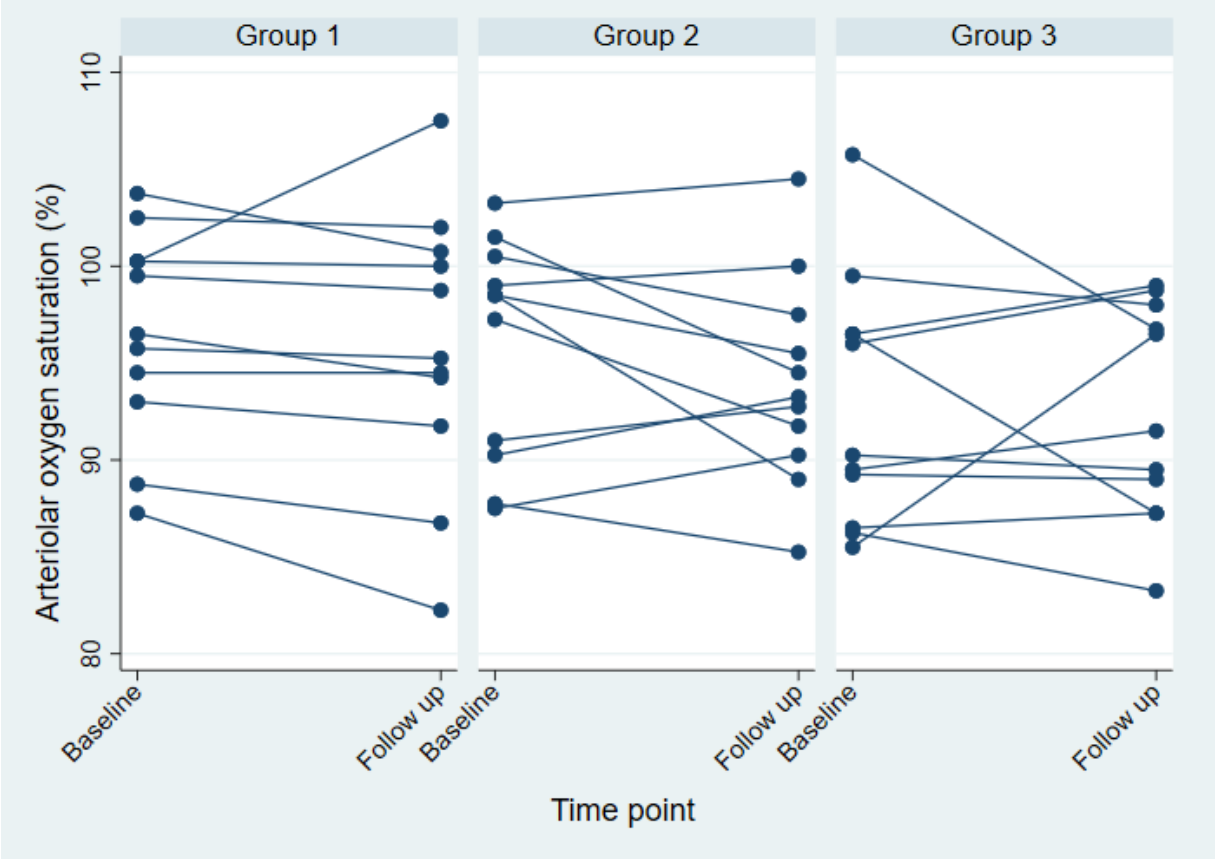


Figure 3:

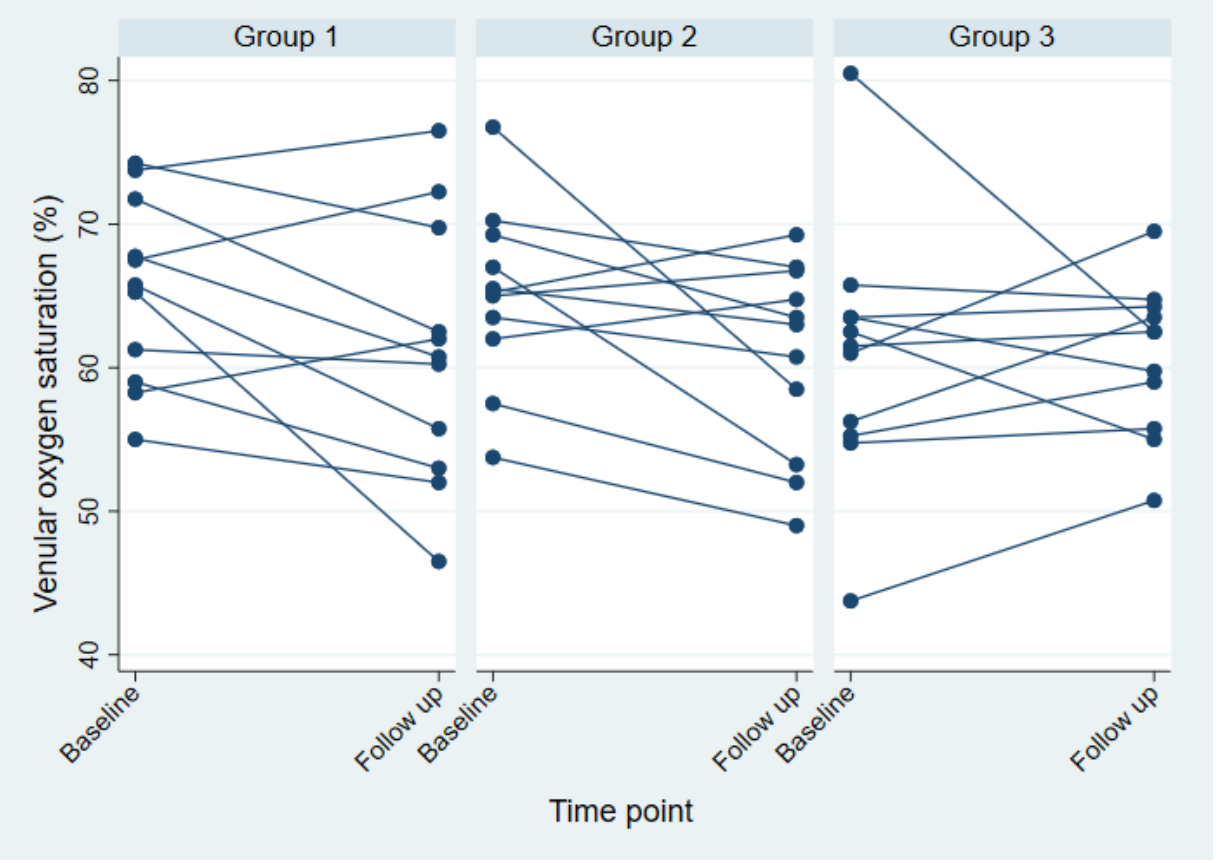


Table 1: Baseline characteristics of the included patients.

\begin{tabular}{|l|c|c|c|c|}
\hline Baseline characteristics & P-value \\
\hline Groups according to no. of laser spots & $<1500$ & $1500-2000$ & $>2000$ & \\
\hline No. of eyes (n) & 10 & 11 & 12 & \\
\hline Age (years) & $53.5 \pm 13.0$ & $52.0 \pm 12.0$ & $54.5 \pm 18.0$ & 0.78 \\
\hline Sex, male (\%) & $60.0 \%$ & $55.0 \%$ & $58.0 \%$ & 0.97 \\
\hline Ethnicity, caucasian (\%) & $100.0 \%$ & $91.0 \%$ & $100 \%$ & 0.36 \\
\hline Body mass index (kg/m ( ) $^{\text {( })}$ & $29.2 \pm 10.6$ & $26.1 \pm 7.2$ & $31.4 \pm 6.6$ & 0.58 \\
\hline Diabetes, type 1 (\%) & $40.0 \%$ & $55.0 \%$ & $42.0 \%$ & 0.76 \\
\hline Diabetes duration (years) & $22.0 \pm 16.0$ & $17.0 \pm 23.0$ & $20.5 \pm 17.0$ & 0.87 \\
\hline Central retinal thickness ( $\mu$ m) & $229.0 \pm 34.0$ & $229.0 \pm 68.0$ & $257.5 \pm 49.5$ & 0.74 \\
\hline Best corrected visual acuity (ETDRS) & $85.0 \pm 4.0$ & $83.0 \pm 8.0$ & $84.5 \pm 8.5$ & 0.41 \\
\hline Systolic blood pressure (mmHg) & $158.0 \pm 26.0$ & $152.0 \pm 38.0$ & $146.0 \pm 19.5$ & 0.36 \\
\hline Diastolic blood pressure (mmHg) & $93.5 \pm 9.0$ & $85.0 \pm 21.0$ & $89.0 \pm 10.0$ & 0.23 \\
\hline HbA1c (mmol/mol) & $61.5 \pm 14.0$ & $65.0 \pm 15.0$ & $71.5 \pm 20.5$ & 0.87 \\
\hline
\end{tabular}

Ba seline characteristics of patients included in the s tudy. Da ta a re presented as median with interquartile range. 
Table 2: Global retinal venular and arteriolar oxygen saturation at baseline and month 6 according to treatment groups

\begin{tabular}{|c|c|c|c|c|c|c|c|c|c|c|c|c|}
\hline \multirow[b]{2}{*}{$\begin{array}{l}\text { No. of laser } \\
\text { spots }\end{array}$} & \multirow[b]{2}{*}{$\mathrm{n}$} & \multicolumn{2}{|c|}{ Image quality } & \multicolumn{3}{|c|}{ Retinal arteriolar oxygen saturation } & \multicolumn{3}{|c|}{$\begin{array}{c}\text { Retinal venular oxygen } \\
\text { saturation }\end{array}$} & \multicolumn{3}{|c|}{ Retinal arterio-venous difference } \\
\hline & & $\mathrm{BL}$ & M6 & $\mathrm{BL}$ & M6 & $\begin{array}{c}\mathrm{P}- \\
\text { value }\end{array}$ & $\mathrm{BL}$ & M6 & P-value & $\mathrm{BL}$ & M6 & P-value \\
\hline$<1500$ & 10 & $7.6 \pm 0.2$ & $7.3 \pm 0.2$ & $96.2 \pm 7.3$ & $94.9 \pm 8.3$ & 0.57 & $65.5 \pm 8.8$ & $60.5 \pm 9.5$ & $0.04^{*}$ & $31.9 \pm 5.8$ & $35.9 \pm 13.0$ & $0.03^{*}$ \\
\hline $1500-2000$ & 11 & $7.1 \pm 0.2$ & $7.2 \pm 0.3$ & $98.5 \pm 10.3$ & $93.3 \pm 7.3$ & 0.16 & $65.3 \pm 7.3$ & $63.0 \pm 13.5$ & $0.04^{*}$ & $31.3 \pm 9.3$ & $33.0 \pm 9.3$ & 0.15 \\
\hline$>2000$ & 12 & $7.7 \pm 0.2$ & $7.3 \pm 0.2$ & $93.2 \pm 10.1$ & $94.0 \pm 10.2$ & 0.75 & $62.0 \pm 8.9$ & $62.5 \pm 7.1$ & 0.97 & $28.4 \pm 13.0$ & $30.3 \pm 10.4$ & 0.84 \\
\hline P-value & & 0.50 & 0.85 & 0.43 & 0.49 & & 0.41 & 0.71 & & 0.86 & 0.49 & \\
\hline
\end{tabular}

Retinal ve nular and arteriolar oxygen saturation and arteorio-venous difference at baseline (BL) compared to month 6 follow up (M6). The patients a re divided into three groups according to the number of laser s pots ap plied during panretinal photocoagulation: group 1 (<1500 s pots), group 2 (1500-2000 s pots) a nd group 3 ( $>2000$ s pots). Data a re presented as median with interquartile range. *Statistically significant $p<0.05$. 
Table 3: Retinal arteriolar and venular diameter at baseline and month 6 according to treatment groups

\begin{tabular}{|l|c|c|c|c|c|c|c|}
\hline \multicolumn{2}{|c|}{} & \multicolumn{3}{|c|}{ Retinal arteriolar diameter } & \multicolumn{3}{c|}{ Retinal venular diameter } \\
\hline No. of laser spots & $\mathrm{n}$ & $\mathrm{BL}$ & $\mathrm{M} 6$ & $\mathrm{P}$-value & $\mathrm{BL}$ & $\mathrm{M} 6$ & P-value \\
& & & & & & & \\
\hline$<1500$ & 10 & $122.75 \pm 25.3$ & $111.0 \pm 17.3$ & 0.19 & $174.5 \pm 15.3$ & $165.1 \pm 28.7$ & $0.01^{*}$ \\
\hline $1500-2000$ & 11 & $123.8 \pm 17.0$ & $122.5 \pm 19.8$ & 0.13 & $168.3 \pm 25.0$ & $166.8 \pm 26.5$ & 0.45 \\
\hline$>2000$ & 12 & $124.0 \pm 26.0$ & $124.0 \pm 15.5$ & 0.30 & $167.8 \pm 34.3$ & $163.2 \pm 35.1$ & 0.81 \\
\hline P-value & & 0.60 & 0.31 & & 0.84 & 0.95 & \\
\hline
\end{tabular}

Retinal ve nular and arteriolar diameter at baseline (BL) compared to month 6 follow up (M6). The patients a re divided into th ree groups according to the number of laser spots applied during panretinal photocoagulation: group 1 (<1500 s pots), group 2 (15002000 s pots) and group 3 (>2000 spots). Data are presented as median with interquartile ra nge. *Statistically significant $p<0.05$. 
Table 4: Focal retinal venular and arteriolar oxygen saturation at baseline and month 6 according to groups in treated quadrants

\begin{tabular}{|l|c|c|c|c|c|c|c|}
\hline \multicolumn{2}{|l|}{} & \multicolumn{2}{l|}{ Retinal arteriolar oxygen saturation } & \multicolumn{4}{l|}{ Retinal venular oxygen saturation } \\
\hline No. of laser spots & $\mathrm{n}$ & $\mathrm{BL}$ & $\mathrm{M} 6$ & $\mathrm{P}$-value & $\mathrm{BL}$ & $\mathrm{M} 6$ & P-value \\
& & & & & & & \\
\hline$<1500$ & 10 & $96.8 \pm 14.0$ & $94.5 \pm 13.0$ & 0.89 & $61.5 \pm 10.5$ & $60.3 \pm 10.0$ & 0.69 \\
\hline $1500-2000$ & 8 & $100.0 \pm 7.5$ & $94.0 \pm 9.6$ & 0.08 & $67.5 \pm 13.3$ & $61.5 \pm 8.8$ & $0.04^{*}$ \\
\hline$>2000$ & 12 & $93.5 \pm 8.0$ & $94.5 \pm 9.0$ & 0.63 & $64.5 \pm 13.0$ & $64.0 \pm 6.0$ & 0.98 \\
\hline P-value & & 0.49 & 0.79 & & 0.51 & 0.84 & \\
\hline
\end{tabular}

Retinal ve nular and arteriolar oxygen saturation located to the quadrants of the retina with neovascularization before and af ter la ser treatment. Pa tients with neovascularization in four and three quadrants was excluded from this analysis. The patients a re divided into three groups a ccording to the number of laser spots applied as panretinal photocoagulation: group 1 ( $<1500$ spots ), group 2 (1500-2000 s pots) and group 3 (>2000 s pots). Data are presented as median with interquartile ra nge. *Statistically significant $\mathrm{p}<0.05$. 